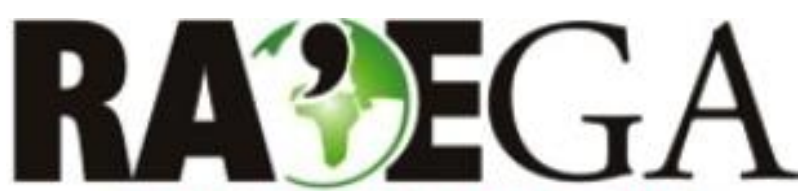

O ESPACCO GEOGRÁFICO EM ANÁLISE

\title{
MATRIZ DE TRANSIÇÃO NA DETECÇÃO DAS MUDANÇAS DO USO E OCUPAÇÃO DO SOLO: ESTUDO DE CASO DO CENTRO EDUCACIONAL UNIFICADO DA PAZ - ZONA NORTE DE SÃO PAULO
}

\section{TRANSITION MATRIX IN THE DETECTION OF LAND USE AND LAND COVER CHANGES: CASE STUDY OF EDUCATIONAL UNIFIED CENTER - NORTH ZONE OF SAO PAULO}

\section{RESUMO}

Pedro Bueno Rocha Campos ${ }^{1}$, Alfredo Pereira de Queiroz Filho ${ }^{2}$

O trabalho apresenta uma análise das modificações do uso e ocupação do solo do entorno do Centro Educacional Unificado (CEU) da Paz, entre 2002 e 2010. Este equipamento público, construído em 2004, está localizado na zona norte do município de São Paulo - SP, considerada periférica e caracterizada pela ocorrência de Assentamentos Urbanos Precários (AUP). A classificação de imagens de 2002, 2004, 2007 e 2010 foi usada para gerar matrizes de transição, para cada intervalo de tempo. Os resultados indicam que o crescimento total de AUP foi de $40,8 \%$, as áreas de mata se reduziram $31,18 \%$ e os outros tipos de domicílios não tiveram crescimento expressivo. Os percentuais parciais indicam que a construção do CEU Paz foi decisiva na expansão dos AUP das adjacências. Até a construção da escola, em 2004, o percentual de crescimento de favelas foi de 3,69\%. Entre 2004 e 2007, as favelas cresceram $21,8 \%$ e $18,31 \%$ das áreas não urbanizadas passaram ser ocupadas por favelas.

PALAVRAS-CHAVE: Matriz de transição, Mudança do uso e ocupação do solo, Centro Educacional Unificado.

\section{ABSTRACT}

The paper presents an analysis of the evolution of urban land use in the vicinity of the Unified Center of Education (UCE) of Paz, between 2002 and 2010. This public facility was built in 2004 and is located in the northern zone of São Paulo - SP. It is considered a peripheral area and is characterized by the occurrence of slums. Images of 2002, 2004, 2007 and 2010 were used for classification of the use and occupation of its surroundings and also to develop a model that identifies the transition matrix of the uses and expresses the dynamic transformations form the growth rate of each class considered. The results indicate that the rate of growth of slums in the period was $40.8 \%$, the forested areas were reduced $31.18 \%$ and other types of households had no significant growth. The partial percentage indicate that the construction of the UCE Paz was fundamental to the AUP expansion in the adjacencies. Until the construction of the equipment, in 2004, the percentage growth of the settlements was 3.69\%. Between 2004 and 2007 , the slum grew $21.8 \%$ and $18.31 \%$ of non-urbanized areas began to be occupied irregularly.

KEY-WORDS: Transition matrix, Land-use change, Unified Center of Education.

Recebido em: 12/07/2016

Aceito em: 30/10/2017

\footnotetext{
${ }^{1}$ Universidade de São Paulo, USP, São Paulo/SP, e-mail: pbrcampos@gmail.com

2 Universidade de São Paulo, USP, São Paulo/SP, e-mail: aqueiroz@usp.br
} 


\section{MATRIZ DE TRANSIÇÃO NA DETECÇÃO DAS MUDANÇAS DO USO E OCUPAÇÃO DO SOLO: ESTUDO DE CASO DO CENTRO EDUCACIONAL UNIFICADO DA PAZ - ZONA NORTE DE SÃO PAULO}

\section{INTRODUÇÃO}

Detecção de mudanças é o processo de mensurar e analisar diferenças entre estados da realidade, em tempos diferentes (SINGH, 1989). A compreensão do processo de mudança do uso e cobertura do solo, no entanto, raramente é obtida por pesquisas isoladas sobre 0 crescimento da população, da pobreza ou da infraestrutura. Boa parte dessas respostas decorre de transformações nas condições econômicas, mediada por fatores institucionais (LAMBINI et al., 2001).

As referências aos modelos de representação quantitativa do ambiente urbano datam do final do século XIX, com destaque para os propostos por Von Thünen, Weber, Christaller e Lösch (MERLIN, 1973). Nas décadas de 1950 e 1960, o aumento da capacidade de processamento de dados possibilitou o aprimoramento dos modelos, que até então analisavam quadros estáticos da realidade, de forma setorizada (ALMEIDA, 2003).

Os modelos espaciais dinâmicos introduzem a dimensão temporal e possibilitam a interação entre diferentes classes de transformações urbanas (WEGENER et al., 1986). Esses modelos se destacam pela capacidade de ampliar os tipos de análises e refinar os parâmetros que expressam as transformações urbanas (LI et al., 2015).

Os modelos dinâmicos surgiram no fim da década 1960, a partir de trabalhos como os de Crecine (1968) e Batty (1971). Suas aplicações variam conforme a complexidade do ambiente urbano. Exemplos de aplicação são os estudos do crescimento urbano (ALMEIDA et al., 2003; LI et al., 2015), saúde pública (GONG et al., 2012), clima urbano (GEORGESCU et al., 2014), detecção de mudanças do uso e cobertura do solo (LU et al., 2004; TURNER et al., 2007; BASSE et al., 2014) ou do sistema viário (BARTHELEMY, 2015).

A comparação de uma sequência cronológica de imagens de satélite, com técnicas de processamento digital, é muito utilizada no mapeamento das mudanças do uso e cobertura do solo (KLEYNHANS et al., 2015; HEGAZY; KALOOP, 2015). No entanto, com o uso da matriz de transição é possível quantificar essas transformações multitemporais com maior exatidão (ALMEIDA et al., 2003; BASSE et al., 2014; LÊ et al., 2015). Para Basse et al., (2014), os quadros estáticos não representam plenamente a dinâmica e as forças motrizes dos processos de transformação urbana. É necessário um elemento de conexão, entre os estados discretos, capaz de detectar a dinâmica intrínseca das transições do uso e cobertura (BATTY, 1999).

Neste artigo, um modelo de detecção de mudanças, baseado em matrizes de transição, foi utilizado para analisar as alterações do uso e ocupação do solo no entorno do Centro Educacional Unificado - CEU, unidade Paz, localizado na zona norte de São Paulo, entre os anos 2002 e 2010.

\section{MATERIAIS E MÉTODOS 2.1 MATRIZ DE TRANSIÇÃO}

A matriz de transição, adaptada dos trabalhos de Almeida et al. (2003), Basse et al. (2014) e Lê et al. (2015), foi utilizada para analisar a detecção de mudanças de uso e ocupação do solo do entorno do CEU Paz. Nesse tipo de modelo, as regras e padrões de transição são associados às variáveis pesquisadas. De acordo com Batty; Cheshire (2011), esse encadeamento de cenários estáticos possibilita a formalização da dinâmica das alterações do uso e ocupação do solo urbano.

De acordo com Dimuro et al. (2002), matrizes de transição podem ser utilizadas na predição de cenários futuros. Calculam a probabilidade de transição das classes analisadas em função da sua variação no tempo. As matrizes utilizadas neste artigo têm como base as cadeias de primeira ordem de Markov. Conforme Almeida et al. (2003), a matriz é calculada pixel a pixel, conforme as fórmulas 1 e 2 abaixo:

$\Delta N^{k l}=\sum_{i} N_{i}^{l}(t+1)-\sum_{i} N_{i}^{k}(t)=N^{l}(t+1)-N^{k}(t)=\sum_{i} \Delta N_{i}^{k l}$

$\mathrm{Na}$ Fórmula 1, $\Delta \mathrm{Nkl}$ representa a variação do uso k para o uso I, no ponto i, entre os universos de células, dos intervalos $\mathrm{N}$ e $\mathrm{N}(\mathrm{t}+$ 1), de análise. A Fórmula 2 representa o cálculo 


\section{MATRIZ DE TRANSIÇÃO NA DETECÇÃO DAS MUDANÇAS DO USO E OCUPAÇÃO DO SOLO: ESTUDO DE CASO DO CENTRO EDUCACIONAL UNIFICADO DA PAZ - ZONA NORTE DE SÃO PAULO}

das probabilidades $\mathrm{Pkl}$, ou seja, as porcentagens de transição entre um uso e outro:

$P^{k l}=\frac{\Delta N^{k l}}{N^{k}(t)}=\frac{\Delta N^{k l}}{\sum \Delta N^{k l}}, e \sum_{l} p^{k l}=1$

A matriz de transição realiza o cálculo das taxas de mudança de cada classe de ocupação através de uma grade de comparação cruzada. Esta matriz indica os valores percentuais de alteração entre uma classe e outra. As taxas são calculadas entre os intervalos de tempo utilizados na classificação do uso e ocupação do solo.

\subsection{CARACTERIZAÇÃO E LOCALIZAÇÃO DA ÁREA DE ESTUDO}

De acordo com Sposati (1996) e Gamba et al. (2012), a Zona Norte do município de São Paulo se caracteriza por um elevado grau de exclusão social e alta vulnerabilidade social, apresentando diferentes níveis de pobreza. Segundo Preteceille (1999), mesmo na pobreza existem desigualdades, principalmente com relação ao espaço de reprodução social, ou seja, a forma de interação com o local onde vivem. A Figura 1 localiza a área de estudo no município de São Paulo.

A região onde foi construído o CEU Paz é considerada periferia da zona norte do município de São Paulo. Está localizado no distrito administrativo da Brasilândia, que possui inúmeros assentamentos urbanos precários e considerável déficit de infraestrutura urbana.

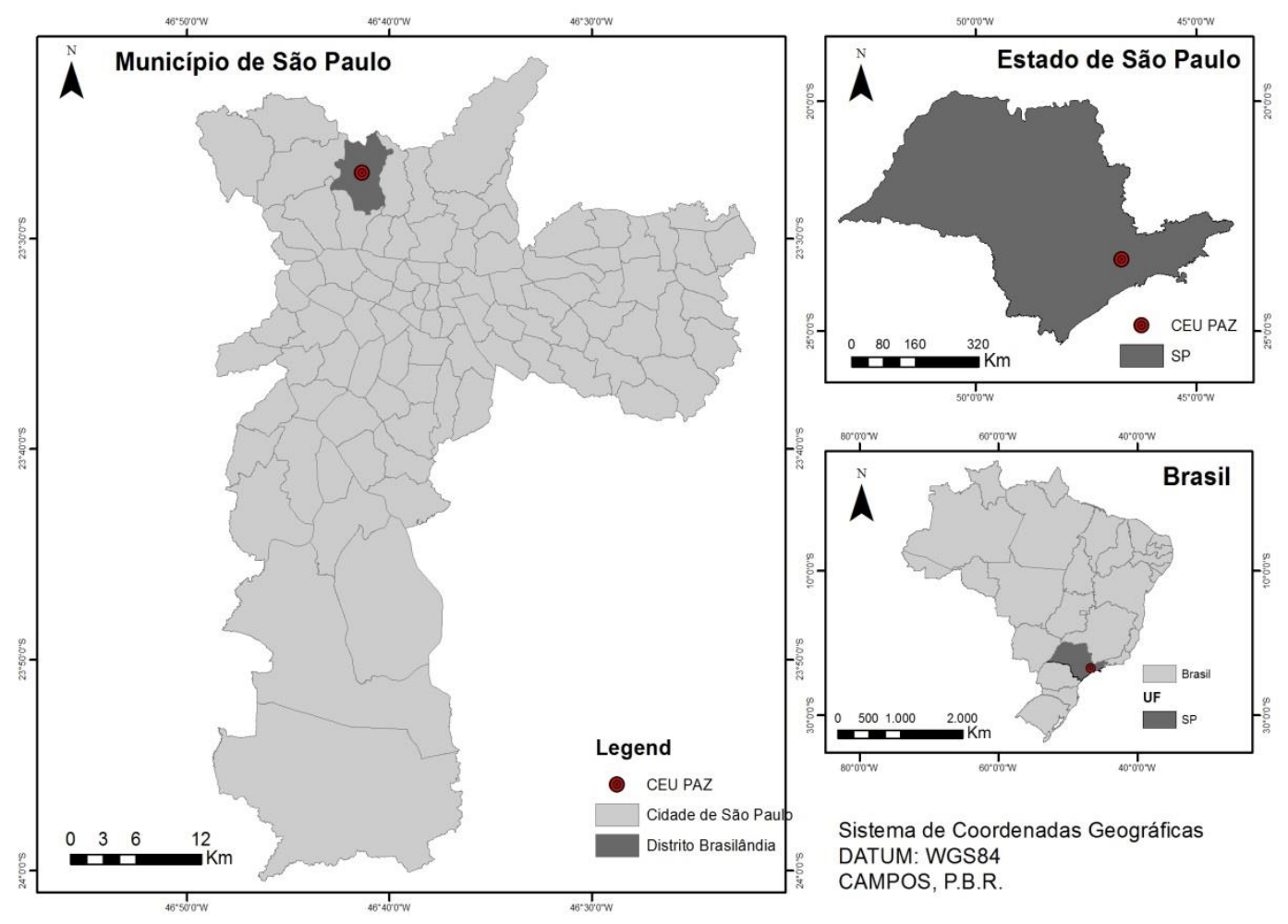

Figura 1 - Localização da Área de Estudo.Fonte: Elaborado pelos autores 
CAMPOS,P.B.R. e QUEIROZ FILHO, A.P.

\section{MATRIZ DE TRANSIÇÃO NA DETECÇÃO DAS MUDANÇAS DO USO E OCUPAÇÃO DO SOLO: ESTUDO DE CASO DO CENTRO EDUCACIONAL UNIFICADO DA PAZ - ZONA NORTE DE SÃO PAULO}

Os CEUs são equipamentos públicos de grande porte e oferecem inúmeros serviços, que entre outros, têm o objetivo de garantir o desenvolvimento da comunidade beneficiada. $\mathrm{O}$ CEU Paz, construído em 2004 (figura 2), tem a finalidade de garantir o acesso da população local aos serviços de educação, esporte, cultura e lazer (PMSP/SME, 2003 e 2004).

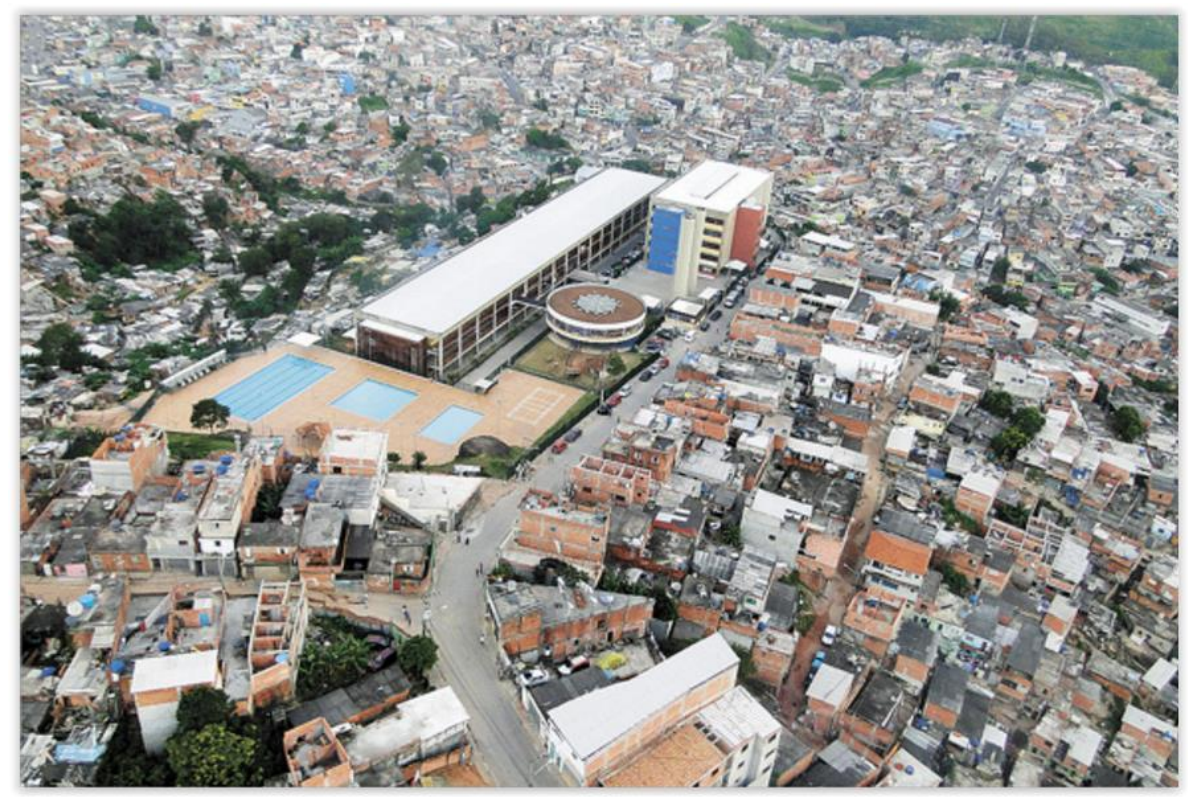

Figura 2 - Fotografia do CEU Paz.Fonte: Gazeta da Zona Norte (2014)

Para Thisse; Wildasin (1992) e Kowarick (1996), intervenções urbanísticas desse porte costumam afetar as estruturas sociais e promover melhorias nos padrões de reprodução e materialização destas relações sociais no espaço.

Entretanto, no caso do CEU da Paz, é possível considerar que não houve somente benefícios, pois ocorreram ocupações irregulares em áreas de preservação ambiental, como a Serra da Cantareira, contrariando diversas diretrizes dos Planos Diretores Estratégico e
Regional e o Plano de Ação Habitacional e Urbana da região (ANGILELI, 2007). Em contrapartida, ainda segundo a autora, esse CEU ajudou a atender a demanda por creche, escola e atividades socioeducativas da população local.

A área de estudo foi delimitada por um raio de aproximadamente 600 metros dos limites do CEU Paz (Figura 3). Foi definida de acordo com a área de influência da unidade e representa a região com maior nível de alteração de tipos de uso no intervalo de tempo da pesquisa. 


\section{MATRIZ DE TRANSIÇÃO NA DETECÇÃO DAS MUDANÇAS DO USO E OCUPAÇÃO DO SOLO: ESTUDO DE CASO DO CENTRO EDUCACIONAL UNIFICADO DA PAZ - ZONA NORTE DE SÃO PAULO}

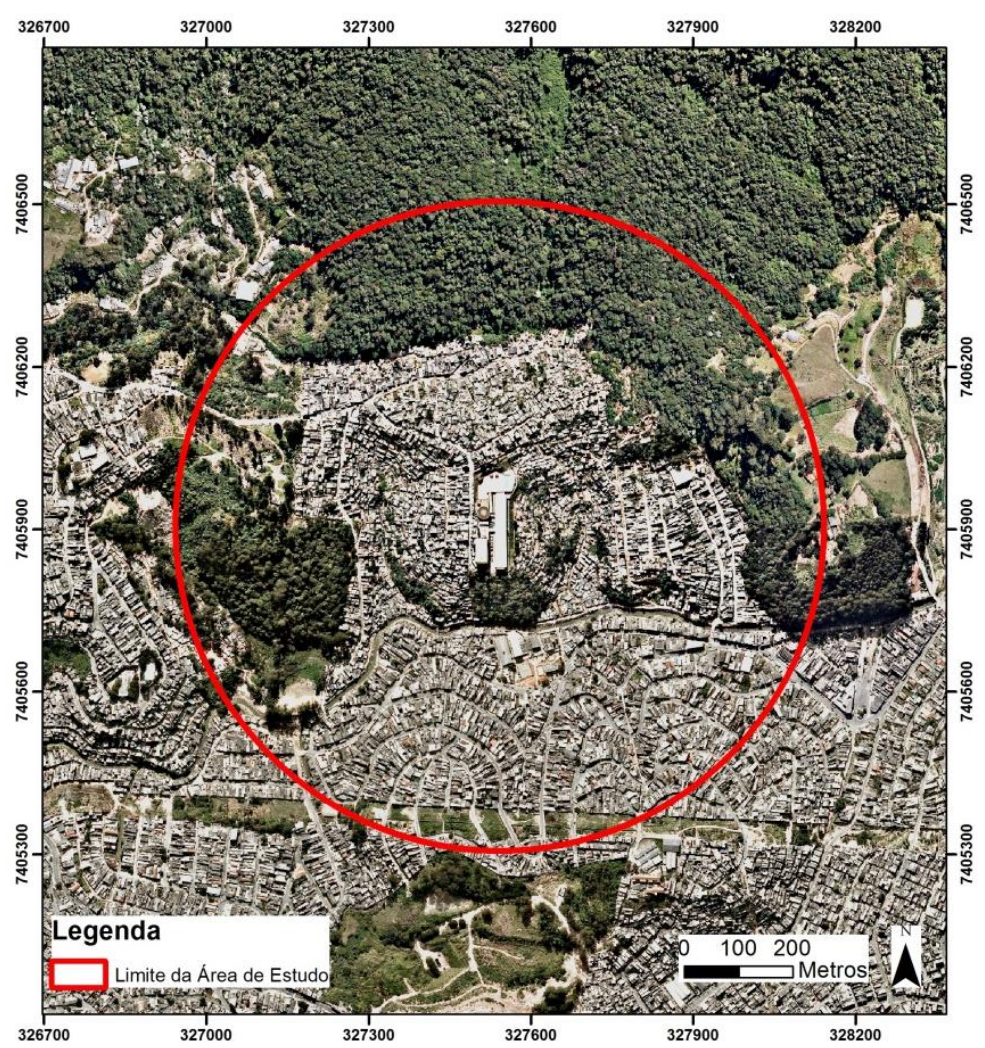

Figura 3 - Área de estudo: entorno do CEU Paz (Sistema de coordenadas UTM, Datum SAD-1969, fuso 23S, Ortofoto de 2010, resolução $1 \mathrm{~m}$ ).

\subsection{PROCEDIMENTOS METODOLÓGICOS E MATERIAIS}

Os procedimentos metodológicos da pesquisa estão ilustrados no fluxograma apresentado na figura 4. As principais etapas foram: 1) classificação do uso e ocupação, a partir de técnicas de interpretação de imagens de satélite e ortofotos; 2) cálculo das matrizes de transição entre os intervalos discretos de tempo; e 3) análise dos padrões de mudança através do encadeamento de transições observado nas matrizes de transição.

\section{Classificação}

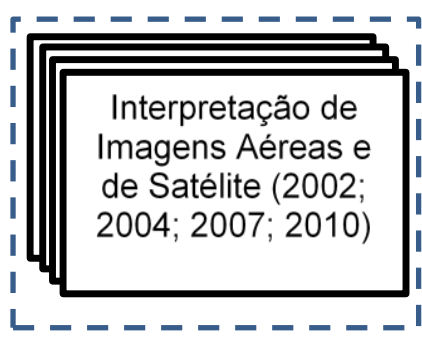

Cálculo Matriz de Transição

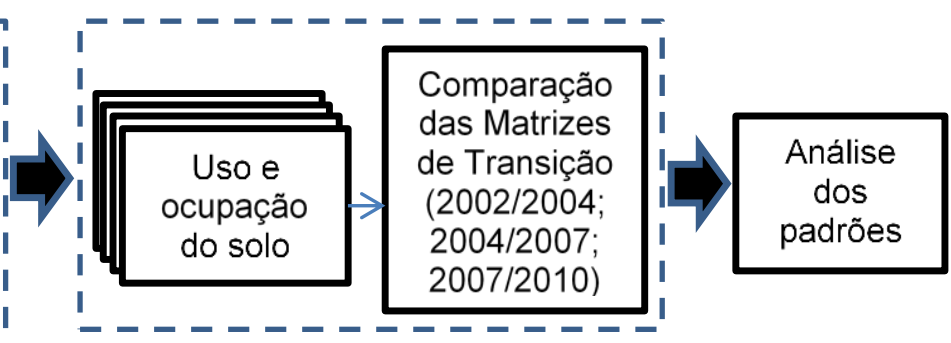

Figura 4 - Fluxograma dos Procedimentos.

Foram classificadas as imagens dos anos de 2002, 2004, 2007 e 2010. As imagens utilizadas estão especificadas na Tabela 1. A classificação foi realizada por interpretação de imagens, de acordo com a metodologia de Kohli et al. (2012). Ela facilita a classificação de usos complexos, como assentamentos urbanos precários, pois utiliza uma abordagem 


\section{MATRIZ DE TRANSIÇÃO NA DETECÇÃO DAS MUDANÇAS DO USO E OCUPAÇÃO DO SOLO: ESTUDO DE CASO DO CENTRO EDUCACIONAL UNIFICADO DA PAZ - ZONA NORTE DE SÃO PAULO}

multiescalar: contexto (localização e vizinhança), telhados).

classe (formato e densidade) e detalhe (vias e

Tabela 1 - Características das imagens da pesquisa

\begin{tabular}{|c|c|c|} 
Imagem & Resolução Espacial & Ano \\
\hline Satélite Ikonos & $1 \mathrm{~m}^{*}$ & 2002 \\
\hline Ortofotos & $0,20 \mathrm{~m}$ & 2004 \\
Ortofotos & $0,60 \mathrm{~m}$ & 2007 \\
\hline Ortofotos & $1 \mathrm{~m}$ & 2010 \\
\hline
\end{tabular}

* Foi utilizada a composição RGB com as bandas vermelha, verde e azul, fusionada com a banda pancromática.

Os critérios utilizados na chave de classificação foram adaptados da metodologia proposta por Kohli et al. (2012) e podem ser descritos como: localização, vizinhança, divisão de quadras, padrão de arruamento e de habitação, densidade de vegetação e habitação, tipo de telhado e ausência de construções. As classes estabelecidas, para todas as imagens utilizadas, foram: Residencial, Assentamento Urbano Precário, Mata, Desocupado, Institucional e CEU Paz (ver chave de classificação da Tabela
2). Além das imagens de sensoriamento remoto, foram usadas informações do sistema municipal de informações georreferenciadas de São Paulo (GEOSAMPA, 2016), em escala compatível com a resolução espacial das imagens, como: rede vetorial de ruas, dados de localização de instituições públicas e privadas, dados vetoriais dos assentamentos urbanos precários do Instituto Brasileiro de Geografia e Estatística, dos anos 2000 e 2010.

Tabela 2 - Chave de Classificação. Elaborado pelos autores, a partir da proposta de Kohli et al. (2012)

\begin{tabular}{|c|l|l|}
\hline Amostra & Classe & \multicolumn{1}{c|}{ Critérios } \\
\hline & Residencial & $\begin{array}{l}\text { Divisão de quadras } \\
\text { Tipos de telhado } \\
\text { Vias asfaltadas }\end{array}$ \\
\hline & Assentamento & $\begin{array}{l}\text { Densidade de casas } \\
\text { Ausência de vias } \\
\text { Tipo telhado }\end{array}$ \\
\hline & Urbano Precário & $\begin{array}{l}\text { Presença de vegetação, } \\
\text { textura granular grossa e } \\
\text { distribuição irregular. }\end{array}$ \\
\hline & Mata & $\begin{array}{l}\text { Ausência de construções } \\
\text { e vegetação }\end{array}$ \\
\hline & Desocupado & $\begin{array}{l}\text { Grandes construções, } \\
\text { como outras escolas e } \\
\text { empresas. }\end{array}$ \\
\hline
\end{tabular}




\section{MATRIZ DE TRANSIÇÃO NA DETECÇÃO DAS MUDANÇAS DO USO E OCUPAÇÃO DO SOLO: ESTUDO DE CASO DO CENTRO EDUCACIONAL UNIFICADO DA PAZ - ZONA NORTE DE SÃO PAULO}

As matrizes de transição foram calculadas no software DINAMICA EGO (software livre, UFMG). Cada matriz representou uma iteração do modelo, que considera o intervalo de tempo entre cada imagem classificada. Foram utilizadas três matrizes de transição para calcular a taxa de mudanças entre as classes de uso e ocupação. Verificou-se a ocorrência de repetições sistemáticas de transições entre cada matriz calculada, que evidencia o encadeamento de sucessões.

\section{RESULTADOS}

Os resultados foram sistematizados em tabelas, mapas e imagens. A Tabela 3 compreende o intervalo entre 2002 e 2004 e representa o período anterior à intervenção urbanística realizada na área de pesquisa. Os cálculos da matriz de transição indicam um crescimento de 10,9\% das áreas desocupadas em substituição às áreas de mata. A taxa de crescimento de assentamentos urbanos precários - AUP foi de 3,69\%. As áreas ocupadas por este tipo de habitação também tiveram origem nas áreas classificadas como mata. Foi possível perceber a construção do CEU Paz, em 2004 (não foram computadas transições para as outras classes de uso).

Tabela 3 - Matriz de transição entre 2002 e 2004.

\begin{tabular}{|l|c|c|c|c|c|c|}
\hline \multicolumn{1}{|c|}{ Uso } & Mata & Residencial & A.U.P & Desocupado & Institucional & CEU \\
\hline Mata & - & - & $3,694 \%$ & $10,913 \%$ & - & $2,756 \%$ \\
\hline Residencial & - & - & - & - & - & - \\
\hline A.U.P & - & - & - & - & - & - \\
\hline Desocupado & - & - & - & - & - & $1,261 \%$ \\
\hline Institucional & - & - & - & - & - & - \\
\hline CEU & - & - & - & - & - & - \\
\hline
\end{tabular}

O período entre 2004 e 2007 (Tabela 4), mostra o primeiro período após a construção do CEU Paz. Fica evidente o início da expansão das AUPs, pois a taxa ultrapassa $21 \%$ nesse intervalo. Entretanto, não é somente a alta taxa de crescimento que torna as informações da tabela interessantes, mas também o encadeamento de transições entre cada período.

Tabela 4 - Matriz de transição entre 2004 e 2007.

\begin{tabular}{|l|c|c|c|c|c|c|}
\hline \multicolumn{1}{|c|}{ Uso } & Mata & Residencial & A.U.P & Desocupado & Institucional & CEU \\
\hline Mata & - & - & $3,490 \%$ & $5,701 \%$ & - & - \\
\hline Residencial & - & - & - & - & - & - \\
\hline A.U.P & - & - & - & - & - & - \\
\hline Desocupado & - & - & $18,313 \%$ & - & - & - \\
\hline Institucional & - & - & - & - & - & - \\
\hline CEU & - & - & - & - & - & - \\
\hline
\end{tabular}

Este encadeamento de transições fica mais claro no período de 2007 a 2010 (Tabela 5). A dinâmica de transição tem início quando, na Tabela 3, nota-se a substituição das áreas de mata por áreas desocupadas, processo que apresenta continuidade nos períodos seguintes. Já na Tabela 4, as áreas desocupadas se 


\section{MATRIZ DE TRANSIÇÃO NA DETECÇÃO DAS MUDANÇAS DO USO E OCUPAÇÃO DO SOLO: ESTUDO DE CASO DO CENTRO EDUCACIONAL UNIFICADO DA PAZ - ZONA NORTE DE SÃO PAULO}

transformaram em AUP. Esta transição se mantém na Tabela 5, e reforça essa tendência.

Tabela 5 - Matriz de transição entre 2007 e 2010.

\begin{tabular}{|l|c|c|c|c|c|c|}
\hline \multicolumn{1}{|c|}{ Uso } & Mata & Residencial & A.U.P & Desocupado & Institucional CEU \\
\hline Mata & - & - & $1,260 \%$ & $3,370 \%$ & - & - \\
\hline Residencial & - & - & - & - & - & - \\
\hline A.U.P & - & - & - & - & - & - \\
\hline Desocupado & - & - & $9,550 \%$ & - & - & - \\
\hline Institucional & - & - & - & - & - & - \\
\hline CEU & - & - & - & - & - & - \\
\hline
\end{tabular}

Este movimento demonstra o avanço de AUP sobre as áreas desocupadas, que tiveram origem em um processo de desmatamento. $\mathrm{Na}$ Figura 5, estão, em sequência cronológica, os mapas que representam a expansão dos AUP no entorno do CEU Paz.
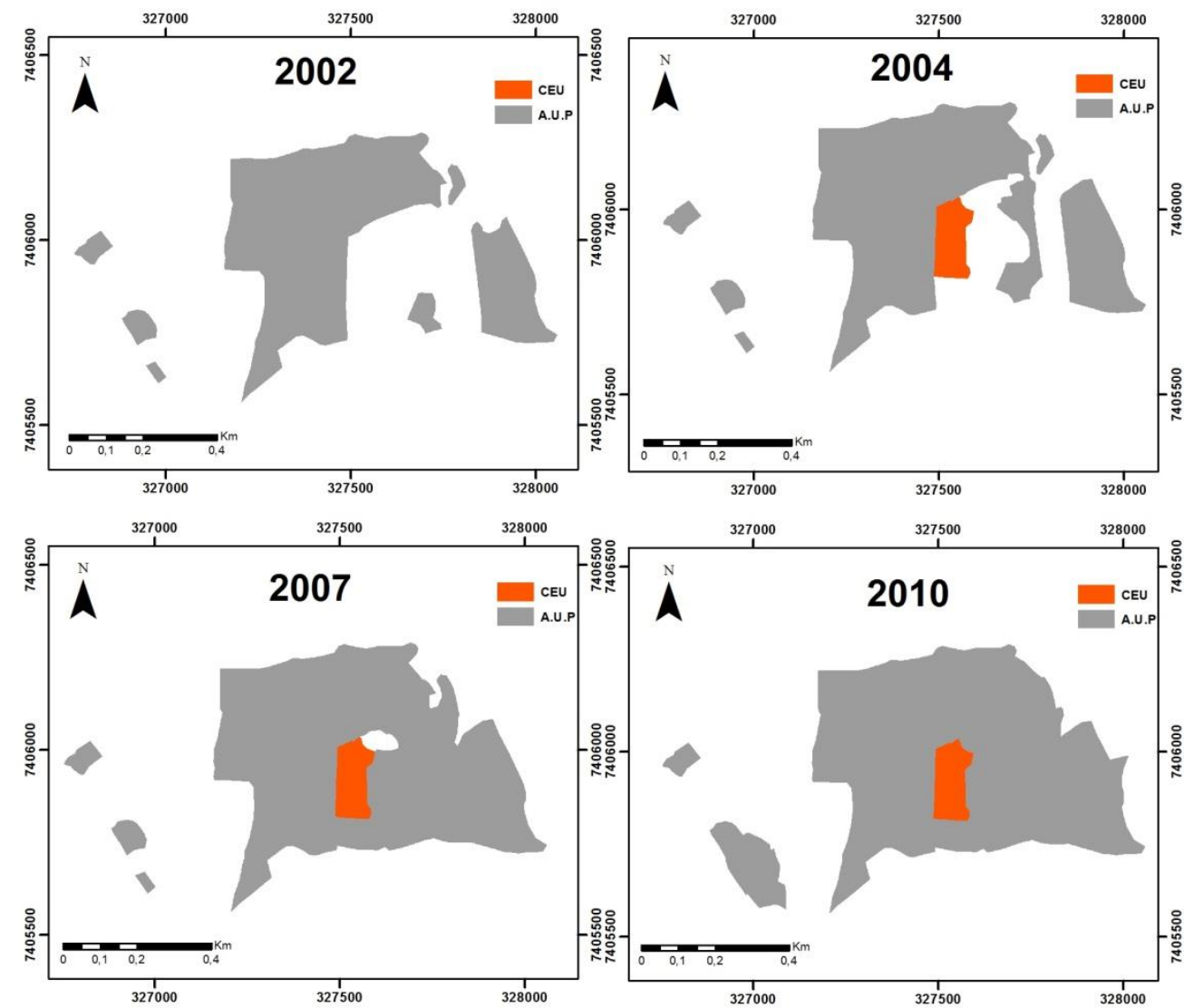

Figura 5 - Mapas da expansão das áreas de assentamentos urbanos precários. (Sistema de coordenadas UTM e Datum SAD-1969, fuso 23S).

Na Figura 6, estão sobrepostas as áreas classificadas como AUP nas respectivas imagens. Nela, é possível visualizar a diminuição das áreas de mata, o aumento da densidade de habitações e o reduzido número de vias de acesso. 


\section{MATRIZ DE TRANSIÇÃO NA DETECÇÃO DAS MUDANÇAS DO USO E OCUPAÇÃO DO SOLO: ESTUDO DE CASO DO CENTRO EDUCACIONAL UNIFICADO DA PAZ - ZONA NORTE DE SÃO PAULO}
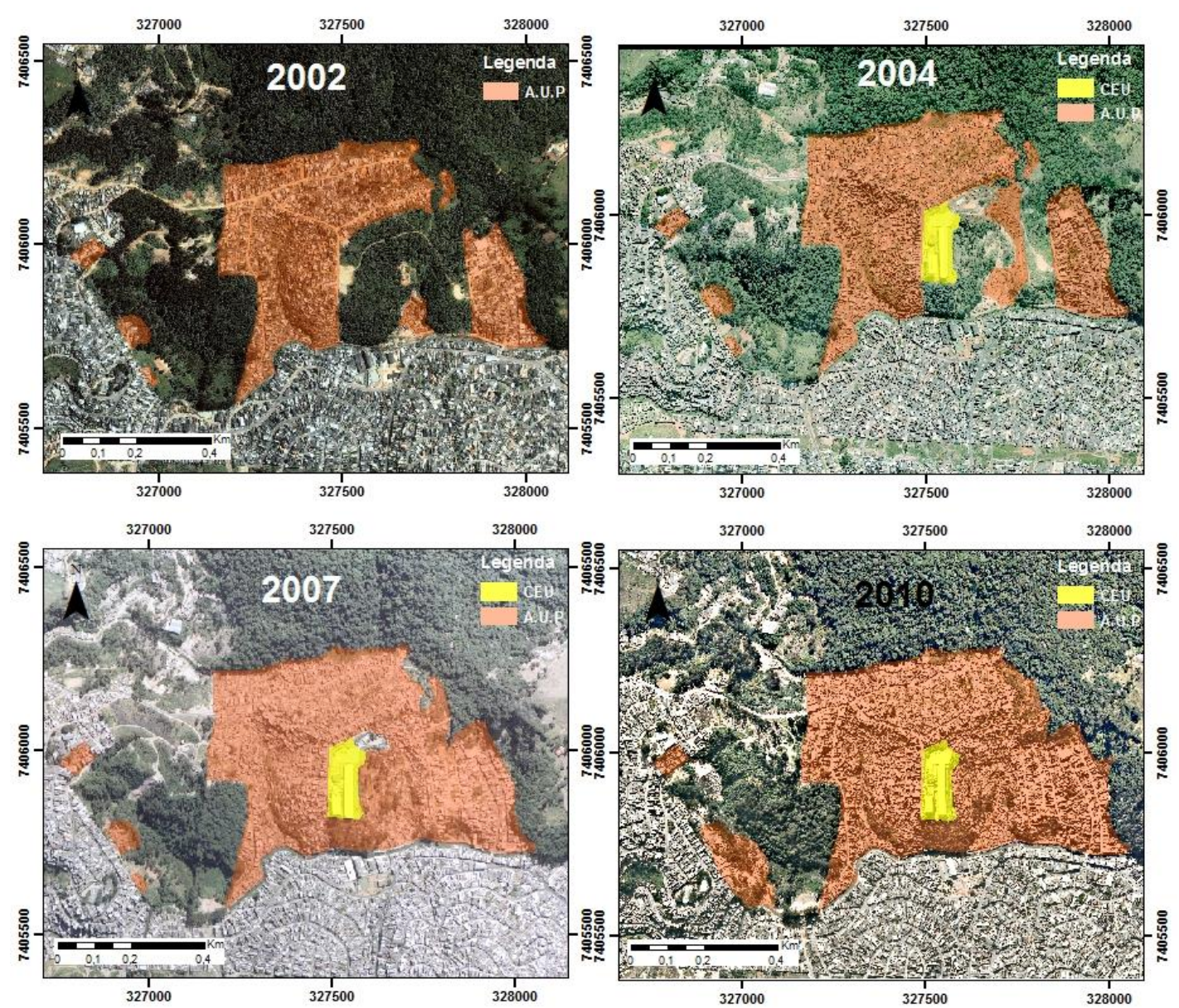

Figura 6 - Sobreposição dos AUP nas imagens (especificadas na Tabela 1. Sistema de coordenadas UTM e Datum SAD-1969, fuso 23S).

Se considerarmos os extremos do intervalo de tempo, de 2002 a 2010, as áreas classificadas como assentamentos urbanos precários aumentaram $40,8 \%$. Outros tipos de domicílios, no entanto, não tiveram crescimento expressivo. As áreas de mata foram reduzidas em $31,18 \%$.

Os valores obtidos em intervalos de tempo menores, no entanto, apontam variações relevantes, particularmente quando se consideram os períodos antes e após a construção do CEU Paz. Até 2004, o percentual de crescimento de AUP foi de $3,69 \%$ e, entre 2004 e 2007 , as AUP cresceram $21,8 \%$ e $18,31 \%$ das áreas não urbanizadas passaram ser ocupadas por AUP.
Os Gráficos 1 e 2 representam a diferença entre os períodos analisados, de 2002 até 2010 e os respectivos intervalos (2002 a 2004, 2004 a 2007 e 2007 a 2010). O Gráfico 1 mostra as divergências, em metros quadrados, das áreas classificadas como AUP. A linha azul representa a taxa de crescimento de cada intervalo e, a linha vermelha, indica o crescimento estimado de cada intervalo no período analisado, considerando uma taxa contínua de crescimento entre 2002 e 2010. 


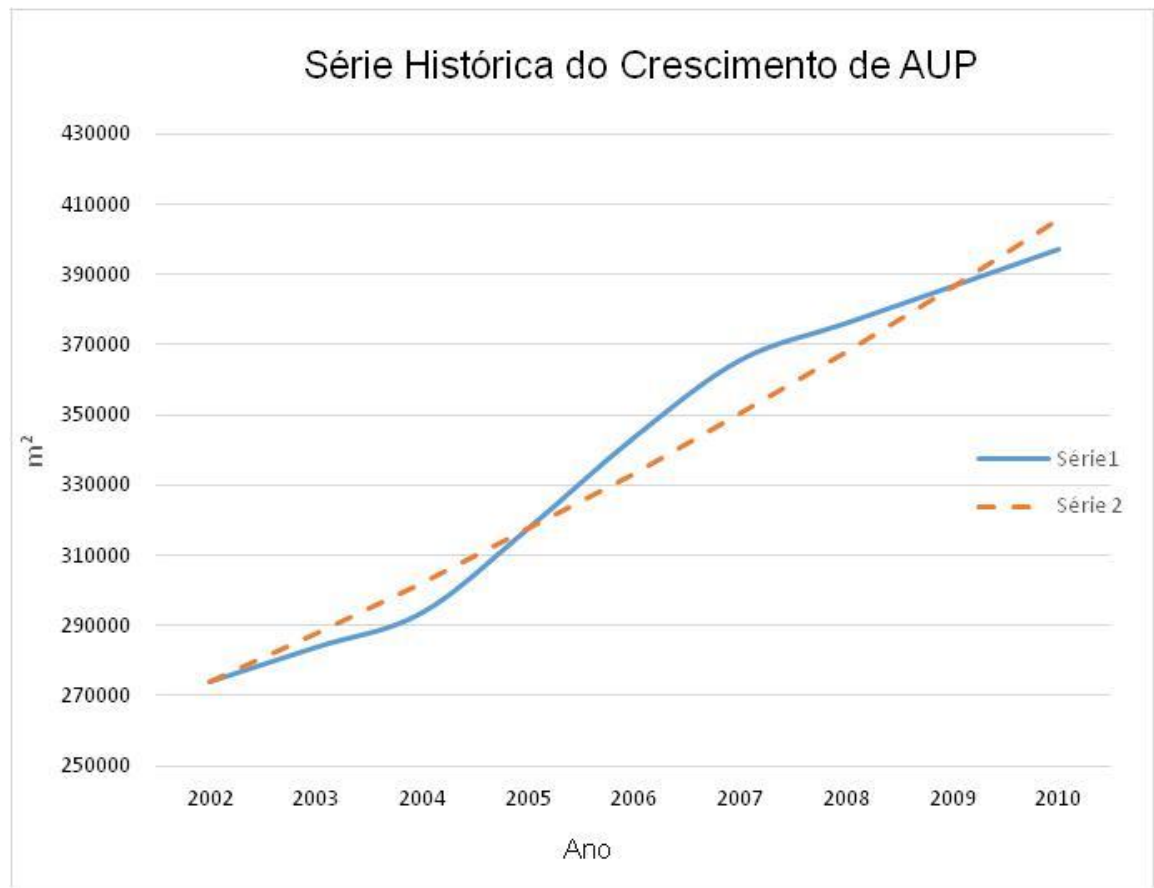

Gráfico 1 - Comparação entre as formas de análise do crescimento de AUP.

Nota-se uma variação na função de crescimento (linha azul), entre os anos 2004 e 2007, enquanto que as inclinações dos segmentos formados entre 2002 a 2004 e 2007 a 2010 são semelhantes. Este fato indica um ponto de ruptura em 2004 e uma estabilização em 2007. Essa interrupção pode ser observada de outra forma no Gráfico 2, que expressa a taxa relativa anual de crescimento dos AUP.

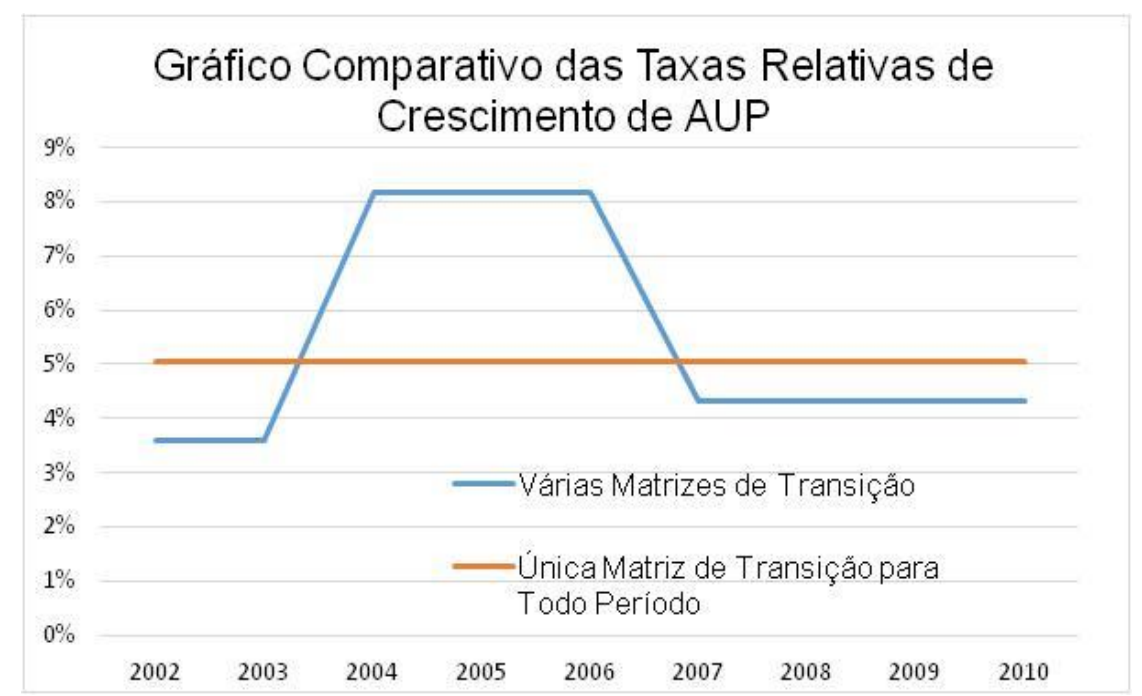

Gráfico 2 - Comparação das taxas relativas de crescimento de AUP.

O mesmo comportamento foi observado nas transições das outras classes. A classe Mata apresentou uma tendência de desmatamento, redução de 17,3\%, no período anterior à construção do CEU Paz, principalmente na transição para áreas desocupadas. Após a implantação do equipamento urbano, a tendência ao desmatamento diminuiu para $9 \%$, entre 2004-2007, e 4\% entre 2007-2010. Em contrapartida, foi observado um intenso processo 


\section{MATRIZ DE TRANSIÇÃO NA DETECÇÃO DAS MUDANÇAS DO USO E OCUPAÇÃO DO SOLO: ESTUDO DE CASO DO CENTRO EDUCACIONAL UNIFICADO DA PAZ - ZONA NORTE DE SÃO PAULO}

de ocupação irregular das áreas desmatadas, que tiveram origem no período entre 2002-2004, como demonstrado pelas taxas de transição de áreas Desocupadas para AUP, de 18\% entre 20042007 e 9\% entre 2007-2010. Esse fato ajuda a ratificar o encadeamento de transições de uso e ocupação do solo, ilustrado nas Tabelas 3, 4 e 5.

\section{DISCUSSÃO}

Os resultados produzidos pelos períodos mais curtos de tempo foram mais representativos quando comparados com os do intervalo mais longo. Para o propósito da pesquisa, o encadeamento das transições discriminou melhor o comportamento do fenômeno. O cálculo de uma matriz transição entre cada intervalo (2002 a 2004, 2004 a 2007 e 2007 a 2010), favoreceu a compreensão dos picos de ocupação do entorno do CEU da Paz.

De outra forma, acredita-se que não seria possível caracterizar o impacto da construção do CEU Paz. Nota-se a variação de 3,69\% (2002-2004) para 21,8\% (2004-2010) no crescimento da área inicial de assentamentos precários, respectivamente antes e após a obra. Caso a análise dos dados fosse feita em uma única matriz de transição, considerando todo o intervalo de 2002 a 2010, o percentual total de crescimento de assentamentos precários, de $40,8 \%$, não discriminaria o período de maior intensidade de expansão e, portanto, não permitiria postular uma relação de causa e efeito (construção/ocupação).

Do ponto de vista da modelagem, pondera-se que a expressiva expansão dos AUP, bem como a modificação da dinâmica de transição das outras classes de uso, após a construção do CEU Paz, caracteriza o que Batty (1999) denominou de vetor de indução. Também corrobora sua afirmação posterior de que quanto maior for a dinâmica do modelo, melhor a capacidade de inferência de dados, que possibilitam a interligação de intervalos discretos de tempo (BATTY, 2012).

Também poderia se estabelecer uma relação com a pesquisa de Feitosa et al. (2011), cujo modelo considera as políticas e os investimentos públicos em infraestrutura como um fator de indução do processo de migração intraurbana. Da mesma forma, à de Zhang et al. (2011), que pesquisaram um modelo de expansão urbana de Xangai, na qual o direcionamento da expansão urbana ocorre em função da priorização de investimentos em determinada área.

No caso do CEU Paz, os resultados mostraram uma ruptura na tendência de crescimento dos usos. Foi possível inferir uma relação entre a construção do CEU e a dinâmica da modificação dos usos. Os indícios são de concentração do crescimento urbano em período e área circunscritos ao CEU Paz.

Em decorrência, esse equipamento público poderia ser caracterizado como um polo indutor que, para Soares-Filho (2006), atua direta e indiretamente na mudança da configuração do ambiente urbano. Ressalta-se, entretanto, que após a consolidação do fenômeno originado ou induzido pelo polo, é provável que ocorra uma retroalimentação de fomento, ou seja, o seu próprio crescimento passa a interferir no seu processo de expansão.

Angileli e Sandeville (2012) reforçam a hipótese de o CEU Paz ter funcionado como um polo atrativo na região. Esta atratividade pode estar relacionada com a possibilidade do aumento de empregos diretos/indiretos, valorização do lote ocupado e o crescimento do comércio informal no entorno do equipamento público, questões que, conforme Roy et al. (2014), estão intrinsecamente relacionadas ao crescimento dos AUP.

Outro aspecto que pode ser considerado na análise da expansão dos AUP foi a falta de integração do CEU com a infraestrutura urbana do entorno. Pacheco (2011) afirma que após a construção do CEU Paz, poucas ruas do entorno foram pavimentadas e que a capacidade do transporte público local se manteve semelhante, alheio ao crescimento populacional.

Os assentamentos precários das imagens foram também comparados com os dados dos censos demográficos dos anos 1991, 2000 e 2010 do Instituto Brasileiro de Geografia e Estatística - 


\section{MATRIZ DE TRANSIÇÃO NA DETECÇÃO DAS MUDANÇAS DO USO E OCUPAÇÃO DO SOLO: ESTUDO DE CASO DO CENTRO EDUCACIONAL UNIFICADO DA PAZ - ZONA NORTE DE SÃO PAULO}

IBGE. Observou-se, contudo, que muitas áreas de AUP não foram registradas pelos censos. Nessas bases de dados, não ocorreu variação significativa entre 1991 e 2000. Já o aumento identificado entre 2000 e 2010, ocorreu em áreas já existentes na primeira data, porém não classificadas como AUP. Por essa razão, não foi possível identificar o impacto da intervenção urbanística considerada nesta pesquisa.

\section{CONSIDERAÇÕES FINAIS}

A taxa de crescimento de assentamentos urbanos precários, de 2002 a 2010, foi de 40,8\%, as áreas de mata se reduziram $31,18 \%$ e os outros tipos de domicílios não tiveram crescimento expressivo. Até a construção do CEU Paz, em 2004, o percentual de crescimento de favelas foi de 3,69\%. Entre 2004 e 2007, as favelas cresceram $21,8 \%$ e $18,31 \%$ das áreas não urbanizadas passaram a ser ocupadas por favelas. O processo de desmatamento, no entanto, registrou uma redução para os períodos após a construção do CEU.

A técnica de matrizes de transição permitiu a identificação de pontos de ruptura nas funções de tendência para todos os usos que tiveram transição no período de análise. As variações encontradas indicam que a construção do CEU Paz influenciou a dinâmica de transformação do uso e ocupação do seu entorno.

Através de modelos preditivos, será possível indicar sob quais condições espaciais este tipo de intervenção urbanística pode influenciar as transformações do uso e ocupação do solo. Estes padrões, se utilizados como regras em modelos baseados em autômatos celulares, também poderiam contribuir para avaliar os eventuais efeitos colaterais de variados tipos de polos indutores, como o que foi identificado nesta pesquisa.

\section{REFERÊNCIAS}

ALMEIDA, C. M.; MONTEIRO, A. M. V.; CÂMARA G. Stochastic cellular automata modeling of urban land use dynamics: empirical development and estimation. Computers, Environment and
Urban Systems, New York, v. 27, n. 5, p. 481-509. 2003.

ALMEIDA, C. M. Modelagem da Dinâmica Espacial como uma Ferramenta Auxiliar ao Planejamento: Simulação de Mudanças de Uso da Terra em Áreas Urbanas para as Cidades de Bauru e Piracicaba (SP), Brasil. Brasil. Tese (Doutorado) Sensoriamento Remoto. Instituto Nacional de Pesquisas Espaciais. 2003.

ANGILELI, C. M. M. M.; SANDEVILLE, E. Uma Experiência de estudos Colaborativos de Paisagens na Brasilândia. Paisagem e Ambiente, São Paulo, v. 30, p. 3, 2012.

ANGILELI, Cecilia Maria de Morais Machado. Paisagem revelada no cotidiano da periferia: Distrito de Brasilândia, Zona Norte do Município de São Paulo. 2007. Tese de Doutorado. Universidade de São Paulo.

BARTHELEMY, M. From paths to blocks: New measures for street patterns. Environment and Planning B: Planning and Design, p. $0265813515599982,2015$.

BASSE, R. M.; OMRANI, H.; CHARIF, O.; GERBER, P.; BÓDIS, K. Land use changes modelling using advanced methods: cellular automata and artificial neural networks. The spatial and explicit representation of land cover dynamics at the cross-border region scale. Applied Geography, v. 53, p. 160-171, 2014.

BATTY, M. Modelling cities as dynamic systems. Nature, v. 231, p. 425-428, 1971.

BATTY, M.; XIE, Y.; SUN, Z. Modeling urban dynamics through GIS-based cellular automata. Computers, environment and urban systems, v. 23, n. 3, p. 205-233, 1999.

BATTY, M; CHESHIRE, J. Cities as flows, cities of flows. Environment and Planning B: Planning and Design, v. 38, n. 2, p. 195-196, 2011.

BATTY, M. A generic framework for computational spatial modelling. In: Agent-based models of geographical systems. Springer Netherlands, p. 19-50, 2012

CRECINE, John P. A dynamic model of urban structure. Santa Monica, RAND Corporation, 1968.

DIMURO, G. P.; REISER, R. H.; COSTA, A. C.; SOUZA, P. L. R. Modelos de Markov e Aplicações. 


\section{MATRIZ DE TRANSIÇÃO NA DETECÇÃO DAS MUDANÇAS DO USO E OCUPAÇÃO DO SOLO: ESTUDO DE CASO DO CENTRO EDUCACIONAL UNIFICADO DA PAZ - ZONA NORTE DE SÃO PAULO}

VI Oficina de Inteligência Artificial, Pelotas: Educat, p. 37-59, 2002.

DINÂMICA EGO, Centro de Sensoriamento Remoto - CSR, Universidade Federal de Minas Gerais, versão 3.1, 2016.

FEITOSA, F. F.; LE, Q. B.; VLEK, P. L. G. Multi-agent simulator for urban segregation (MASUS): A tool to explore alternatives for promoting inclusive cities. Computers, Environment and Urban Systems, v. 35, n. 2, p. 104-115, 2011.

GAMBA, C.; RIBEIRO, W. C. Indicador e avaliação da vulnerabilidade socioambiental no município de São Paulo. Geousp (USP), São Paulo, v. 31, p. 19-31. 2012.

GAZETA DA ZONA NORTE Imagem oblíqua da área de estudo. 2014. Disponível em: <http://www.gazetazn.com.br/index1.asp?bm=m $\& e d=178 \& s=190 \& m a=1272 \& c=0 \& m=0>$. Acesso em: 24 mai 2016.

GEORGESCU, M.; MOREFIELD, P. E.; BIERWAGEN, B. G.; WEAVER, C. P. Urban adaptation can roll back warming of emerging megapolitan regions. Proceedings of the National Academy of Sciences, v. 111, n. 8, p. 2909-2914, 2014.

GEOSAMPA, Secretaria Municipal de Urbanismo e Licenciamento. Prefeitura Municipal de São Paulo. Disponível em: < geosampa.prefeitura.sp.gov.br>. Acesso em: 02 maio 2016.

GONG, P.; LIANG, S.; CARLTON, E. J.; JIANG, Q.; WU, J.; WANG, L.; REMAIS, J. V. Urbanisation and health in China. The Lancet, v. 379, n. 9818, p. 843-852, 2012.

HEGAZY, I. R.; KALOOP, M. R. Monitoring urban growth and land use change detection with GIS and remote sensing techniques in Daqahlia governorate Egypt. International Journal of Sustainable Built Environment, v. 4, n. 1, p. 117$124,2015$.

KLEYNHANS, W.; SALMON, B. P.; OLIVIER, J. C. Detecting settlement expansion in South Africa using a hyper-temporal SAR change detection approach. International Journal of Applied Earth Observation and Geoinformation, v. 42, p. 142149, 2015.

KOHLI, D.; SLIUZAS, R.; KERLE, N.; STEIN, A. An ontology of slums for image-based classification.
Computers, Environment and Urban Systems, Netherland, v. 36, p. 154-163. 2012.

KOWARICK, L. Expoliación urbana, luchas sociales y ciudadanía: retazos de nuestra historia reciente. Estudios sociológicos, p. 729-743, 1996

LAMBIN, E. F. et al. The causes of land-use and land-cover change: moving beyond the myths. Global environmental change, v. 11, n. 4, p. 261269, 2001.

LÊ, T. T. et al. Change detection matrix for multitemporal filtering and change analysis of SAR and PoISAR image time series. ISPRS Journal of Photogrammetry and Remote Sensing, v. 107, p. 64-76, 2015.

LI, X.; GONG, P.; LIANG, L. A 30-year (1984-2013) record of annual urban dynamics of Beijing City derived from Landsat data. Remote Sensing of Environment, v. 166, p. 78-90, 2015.

LU, D.; MAUSEL, P.; BRONDIZIO, E.; MORAN, E. Change detection techniques. International Journal of remote sensing, v. 25, n. 12 , p. $2365-$ 2401, 2004.

MERLIN, Pierre. Méthodes quantitatives et espace urbain. Masson, 1973.

PACHECO, R. T. B. A Política Educacional como Espetáculo: a construção dos centros educacionais unificados em São Paulo. Revista Psicologia Política (Impresso), São Paulo, v. 11, p. 329-343, 2011.

PMSP/SME - Prefeitura Municipal de São Paulo/Secretaria Municipal de Educação. Revista EducAção. PMSP, n. 4. 2003.

PMSP/SME - Prefeitura Municipal de São Paulo/Secretaria Municipal de Educação. Revista EducAção. PMSP, n. 5. 2004.

PRETECEILLE E, VALLADARES L. A desigualdade entre os pobres: favela, favelas. In: HENRIQUES R. (Org.). Desigualdade e pobreza no Brasil. Rio de Janeiro, Instituto de Pesquisa Econômica Aplicada, p. 459-85.1999.

ROY, D.; LEES, M. H.; PALAVALLI, B.; PFEFFER, K.; SLOOT $P$. The Emergence of Slums: $A$ Contemporary View on Simulation Models. Environmental Modelling \& Software, Amsterdam, Elsevier, v. 59, p. 76-90. 2014. SOARES-FILHO, B. S.; NEPSTAD, D. C.; CURRAN, L. M.; CERQUEIRA, G. C.; GARCIA, R. A.; RAMOS, C. 


\section{MATRIZ DE TRANSIÇÃO NA DETECÇÃO DAS MUDANÇAS DO USO E OCUPAÇÃO DO SOLO: ESTUDO DE CASO DO CENTRO EDUCACIONAL UNIFICADO DA PAZ - ZONA NORTE DE SÃO PAULO}

A. Modelling conservation in the Amazon basin. Nature, v. 440, n. 7083, p. 520-523, 2006.

SINGH, Ashbindu. Review article digital change detection techniques using remotely-sensed data. International journal of remote sensing, v. 10, n. 6, p. 989-1003, 1989.

SPOSATI, A. Mapa da exclusão/inclusão social. São Paulo: EDUC/PUC, 1996.

THISSE, J.; WILDASIN, D. E. Public facility location and urban spatial structure: Equilibrium and welfare analysis. Journal of Public Economics, v. 48, n. 1, p. 83-118, 1992.

TURNER, B. L.; LAMBIN, Eric F.; REENBERG, Anette. The emergence of land change science for global environmental change and sustainability. Proceedings of the National Academy of Sciences, v. 104, n. 52, p. 2066620671, 2007.

WEGENER, M.; GNAD, F.; VANNAHME, M. The time escala of urban change. In: Hutchinson, B.; Batty, M. ed. Advances in urban systems modelling. Amsterdam: Elsevier, p. 175-197, 1986.

ZHANG, Qian et al. Simulation and analysis of urban growth scenarios for the Greater Shanghai Area, China. Computers, Environment and Urban Systems, v. 35, n. 2, p. 126-139, 2011 